\title{
Liquid Phase Equilibria of Aqueous Mixtures of Carboxylic Acids (C1 -C4) with Ethylbenzene: Thermodynamic and Mathematical Modeling
}

\author{
S. Laleh Seyed Saadat, ${ }^{\dagger}$ Ali Ghanadzadeh Gilani, ${ }^{+}{ }^{\dagger}$ Hossein Ghanadzadeh Gilani, ${ }^{\ddagger}$ \\ Atefeh Kashef, ${ }^{\dagger}$ and Saeed Fallahi ${ }^{\ddagger}+$ \\ $†$ Department of Chemistry, Faculty of Science and $\neq$ Department of Chemical Engineering, \\ University of Guilan, Rasht, Iran \\ $\neq+$ Young Researchers Club, Shiraz Branch, Islamic Azad University, Shiraz, Iran
}

Table S1 Solubility Curve Data in Mass Fraction $\left(w_{i}\right)$ For $\{$ Water $(1)+$ Carboxylic Acid $(2)+$ Ethylbenzene

(3) $\}$ at $T=298.15 \mathrm{~K}$ and $p=101.32 \mathrm{kPa}$ for the Investigated Systems ${ }^{\text {a }}$

\begin{tabular}{|c|c|c|c|c|c|c|c|}
\hline \multicolumn{2}{|c|}{$\begin{array}{c}\text { water (1) + FA (2) + } \\
\text { ethylbenzene (3) }\end{array}$} & \multicolumn{2}{|c|}{$\begin{array}{c}\text { water (1) + AA (2) + } \\
\text { ethylbenzene (3) }\end{array}$} & \multicolumn{2}{|c|}{$\begin{array}{c}\text { water (1) + PA (2) + } \\
\text { ethylbenzene (3) }\end{array}$} & \multicolumn{2}{|c|}{$\begin{array}{c}\text { water (1) + BA (2) + } \\
\text { ethylbenzene (3) }\end{array}$} \\
\hline$w_{1}$ & $w_{2}$ & $w_{1}$ & $w_{2}$ & $w_{1}$ & $w_{2}$ & $w_{1}$ & $w_{2}$ \\
\hline $0.9997^{b}$ & 0.0000 & $0.9997^{b}$ & 0.0000 & $0.9997^{b}$ & 0.0000 & $0.9997^{b}$ & 0.0000 \\
\hline 0.8879 & 0.1077 & 0.8929 & 0.1035 & 0.8928 & 0.1051 & 0.8896 & 0.1061 \\
\hline 0.8354 & 0.1603 & 0.8391 & 0.1573 & 0.8418 & 0.1560 & 0.8384 & 0.1572 \\
\hline 0.7919 & 0.2015 & 0.7955 & 0.2009 & 0.7842 & 0.2136 & 0.7898 & 0.2058 \\
\hline 0.6915 & 0.2998 & 0.7347 & 0.2618 & 0.7323 & 0.2625 & 0.6961 & 0.2983 \\
\hline 0.5920 & 0.3982 & 0.6455 & 0.3497 & 0.6294 & 0.3600 & 0.5934 & 0.3956 \\
\hline 0.4329 & 0.5566 & 0.5451 & 0.4478 & 0.5877 & 0.3960 & 0.4881 & 0.4870 \\
\hline 0.4001 & 0.5890 & 0.5016 & 0.4902 & 0.4844 & 0.4914 & 0.4340 & 0.5283 \\
\hline 0.2961 & 0.6908 & 0.3914 & 0.5900 & 0.3832 & 0.5734 & 0.3856 & 0.5673 \\
\hline 0.2506 & 0.7344 & 0.2905 & 0.6769 & 0.2757 & 0.6374 & 0.2656 & 0.6091 \\
\hline 0.1965 & 0.7861 & 0.2368 & 0.7018 & 0.2133 & 0.6502 & 0.2097 & 0.6043 \\
\hline 0.1423 & 0.8136 & 0.1853 & 0.7274 & 0.1654 & 0.6544 & 0.1241 & 0.5620 \\
\hline 0.1136 & 0.7854 & 0.0999 & 0.6761 & 0.1000 & 0.6323 & 0.0888 & 0.5450 \\
\hline 0.0735 & 0.6959 & 0.0799 & 0.6456 & 0.0663 & 0.6081 & 0.0646 & 0.5136 \\
\hline 0.0584 & 0.6607 & 0.0618 & 0.6110 & 0.0469 & 0.5287 & 0.0465 & 0.4774 \\
\hline 0.0485 & 0.6197 & 0.0448 & 0.5272 & 0.0282 & 0.4374 & 0.0301 & 0.3923 \\
\hline 0.0334 & 0.5335 & 0.0364 & 0.4782 & 0.0215 & 0.3470 & 0.0240 & 0.3420 \\
\hline 0.0250 & 0.4839 & 0.0278 & 0.4382 & 0.0184 & 0.3108 & 0.0183 & 0.2952 \\
\hline 0.0123 & 0.2998 & 0.0207 & 0.3431 & 0.0114 & 0.2990 & 0.0120 & 0.2497 \\
\hline 0.0074 & 0.2543 & 0.0110 & 0.2552 & 0.0099 & 0.2488 & 0.0073 & 0.2065 \\
\hline 0.0025 & 0.1542 & 0.0030 & 0.1528 & 0.0050 & 0.1499 & 0.0049 & 0.1506 \\
\hline $0.0008^{b}$ & 0.0000 & $0.0008^{b}$ & 0.0000 & $0.0008^{b}$ & 0.0000 & $0.0008^{b}$ & 0.0000 \\
\hline
\end{tabular}

\footnotetext{
${ }^{\mathrm{a}}$ Standard uncertainties $\mathrm{u}$ are $\mathrm{u}(T)=0.01 \mathrm{~K}, \mathrm{u}(p)=0.40 \mathrm{kPa}$, and $\mathrm{u}(\mathrm{w})=0.0050$.

${ }^{b}$ Solubility of binaries Taken from ref 24 and 38 .
} 
Table S2 Experimental Refractive Indices $\left(n_{D}\right)$ As a Function of Water Mass Fraction $\left(\mathbf{w}_{1}\right)$ in the Aqueous Phase Lying on the Solubility Curves at $T=298.15 \mathrm{~K}$ and $p=101.32 \mathrm{kPa}$ for the Investigated Systems ${ }^{\mathrm{a}}$

\begin{tabular}{|c|c|c|}
\hline system & $w_{11}$ & $n_{D}$ \\
\hline \multirow{5}{*}{ water $(1)+$ formic acid (2) + ethylbenzene (3) } & 0.8879 & 1.3374 \\
\hline & 0.8354 & 1.3388 \\
\hline & 0.7919 & 1.3416 \\
\hline & 0.7309 & 1.3441 \\
\hline & 0.6915 & 1.3463 \\
\hline \multirow{9}{*}{ water $(1)+$ acetic acid (2) + ethylbenzene (3) } & 0.6453 & 1.3481 \\
\hline & 0.5920 & 1.3512 \\
\hline & 0.8929 & 1.3408 \\
\hline & 0.8391 & 1.3468 \\
\hline & 0.7955 & 1.3496 \\
\hline & 0.7347 & 1.3522 \\
\hline & 0.6455 & 1.3576 \\
\hline & 0.5451 & 1.3663 \\
\hline & 0.5016 & 1.3683 \\
\hline \multirow{7}{*}{ water $(1)+$ propionic acid $(2)+$ ethylbenzene $(3)$} & 0.8928 & 1.3443 \\
\hline & 0.8418 & 1.3492 \\
\hline & 0.7842 & 1.3552 \\
\hline & 0.7323 & 1.3596 \\
\hline & 0.6294 & 1.3686 \\
\hline & 0.5877 & 1.3719 \\
\hline & 0.4844 & 1.3788 \\
\hline \multirow{7}{*}{ water $(1)+$ butyric acid (2) + ethylbenzene (3) } & 0.8896 & 1.342 \\
\hline & 0.8384 & 1.3509 \\
\hline & 0.7898 & 1.3585 \\
\hline & 0.7441 & 1.3644 \\
\hline & 0.6961 & 1.3715 \\
\hline & 0.6479 & 1.3788 \\
\hline & 0.5934 & 1.3885 \\
\hline
\end{tabular}

${ }^{\mathrm{a}}$ Standard uncertainties $\mathrm{u}$ are $\mathrm{u}(T)=0.01 \mathrm{~K}, \mathrm{u}(p)=0.40 \mathrm{kPa}, \mathrm{u}\left(\mathrm{n}_{\mathrm{D}}\right)=0.0002, \mathrm{u}(w)=0.005$. 
Table S3 Results of the Regression of the Distribution Coefficients for the LSER Equations.

\begin{tabular}{|c|c|c|c|c|c|}
\hline System & $\log D_{2}^{0}$ & $a$ & $b$ & $c$ & $R^{2}$ \\
\hline water (1) + formic acid (2) + ethylbenzene (3) & 1.7068 & 0.00 & -1.51 & -5.14 & 0.727 \\
\hline water $(1)+$ acetic acid $(2)+$ ethylbenzene (3) & 0.9469 & 0.00 & -1.32 & -4.49 & 0.913 \\
\hline water $(1)+$ propionic acid $(2)+$ ethylbenzene $(3)$ & 0.0159 & 0.00 & 0.02 & 0.06 & 0.984 \\
\hline water $(1)+$ butyric acid (2) + ethylbenzene (3) & 0.6941 & 0.00 & 0.51 & 1.75 & 0.935 \\
\hline
\end{tabular}


Table S4 Results of the Regression of the Separation Factors for the LSER Equations.

\begin{tabular}{lccccc}
\hline System & $\log S^{0}$ & $a$ & $b$ & $c$ & $R^{2}$ \\
\hline water $(1)+$ formic acid $(2)+$ ethylbenzene (3) & -4.7355 & 0.00 & 2.22 & 7.53 & 0.937 \\
water $(1)+$ acetic acid $(2)+$ ethylbenzene (3) & -0.1059 & 0.00 & 2.06 & 6.99 & 0.969 \\
water $(1)+$ propionic acid (2) + ethylbenzene (3) & 0.7106 & 0.00 & 2.88 & 9.81 & 0.981 \\
water $(1)+$ butyric acid (2) + ethylbenzene (3) & 0.3850 & 0.00 & 3.12 & 10.60 & 0.960 \\
\hline
\end{tabular}


Table S5 Overall Composition of Liquid-Liquid Equilibrium Data for the Ternary Systems at $T=298.15 \mathrm{~K}$.

\begin{tabular}{ccc}
\hline$z_{1}$ & $z_{2}$ & $z_{3}$ \\
\hline & water $(1)+$ formic acid $(2)+$ ethylbenzene $(3)$ & \\
0.4435 & 0.1116 & 0.4449 \\
0.4007 & 0.1999 & 0.3994 \\
0.3641 & 0.2723 & 0.3636 \\
0.3331 & 0.3339 & 0.3330 \\
0.3071 & 0.3849 & 0.3080 \\
& 0.4287 & 0.2855 \\
0.4458 & water $(1)+$ acetic acid $(2)+$ ethylbenzene $(3)$ & \\
0.3999 & 0.1124 & 0.4434 \\
0.3635 & 0.2001 & 0.4000 \\
0.3334 & 0.2725 & 0.3640 \\
0.3076 & 0.3332 & 0.3334 \\
0.2862 & 0.3848 & 0.3076 \\
& 0.4284 & 0.2854 \\
0.4451 & water $(1)+$ propionic acid $(2)+$ ethylbenzene $(3)$ & \\
0.4002 & 0.1116 & 0.4433 \\
0.3630 & 0.1997 & 0.4001 \\
0.3335 & 0.2703 & 0.3640 \\
0.3079 & 0.3331 & 0.3334 \\
0.2859 & 0.3845 & 0.3076 \\
& 0.4287 & 0.2854 \\
0.4463 & & \\
0.3989 & water (1) + butyric acid (2) + ethylbenzene (3) & \\
0.3622 & 0.1111 & 0.4426 \\
0.3347 & 0.2016 & 0.3995 \\
0.2853 & 0.2742 & 0.3636 \\
& 0.3319 & 0.3334 \\
& 0.3837 & 0.3075 \\
& 0.4292 & 0.2855 \\
\hline
\end{tabular}


Table S6 Calculated GMDH Tie-Line Data in Mass Fraction.

\begin{tabular}{|c|c|c|c|}
\hline$w_{11}$ & $w_{21}$ & $w_{13}$ & $w_{23}$ \\
\hline \multicolumn{4}{|c|}{ water + formic acid +ethylbenzene } \\
\hline 0.711 & 0.312 & 0.000 & 0.000 \\
\hline 0.596 & 0.391 & 0.006 & 0.002 \\
\hline 0.504 & 0.468 & 0.004 & 0.043 \\
\hline 0.431 & 0.542 & 0.002 & 0.060 \\
\hline 0.412 & 0.615 & 0.002 & 0.057 \\
\hline 0.325 & 0.666 & 0.000 & 0.039 \\
\hline \multicolumn{4}{|c|}{ water + acetic acid + ethylbenzene } \\
\hline 0.795 & 0.241 & 0.002 & 0.010 \\
\hline 0.663 & 0.336 & 0.005 & 0.034 \\
\hline 0.579 & 0.408 & 0.006 & 0.057 \\
\hline 0.514 & 0.465 & 0.005 & 0.082 \\
\hline 0.468 & 0.522 & 0.006 & 0.097 \\
\hline 0.391 & 0.562 & 0.010 & 0.118 \\
\hline \multicolumn{4}{|c|}{ water + propionic acid + ethylbenzene } \\
\hline 0.836 & 0.150 & 0.003 & 0.094 \\
\hline 0.774 & 0.226 & 0.006 & 0.169 \\
\hline 0.714 & 0.290 & 0.010 & 0.253 \\
\hline 0.672 & 0.317 & 0.017 & 0.359 \\
\hline 0.632 & 0.357 & 0.031 & 0.460 \\
\hline 0.597 & 0.394 & 0.056 & 0.561 \\
\hline \multicolumn{4}{|c|}{ water + butyric acid +ethylbenzene } \\
\hline 0.925 & 0.060 & 0.008 & 0.156 \\
\hline 0.951 & 0.061 & 0.010 & 0.307 \\
\hline 0.921 & 0.089 & 0.024 & 0.438 \\
\hline 0.896 & 0.072 & 0.043 & 0.411 \\
\hline 0.876 & 0.090 & 0.071 & 0.464 \\
\hline 0.869 & 0.133 & 0.138 & 0.608 \\
\hline
\end{tabular}

